\begin{tabular}{|c|c|}
\hline \multirow{3}{*}{ 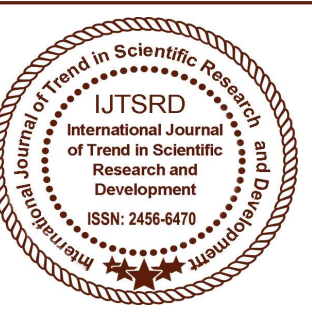 } & $\begin{array}{l}\text { International Journal of Trend in Scientific } \\
\text { Research and Development (IJTSRD) }\end{array}$ \\
\hline & International Open Access Journal \\
\hline & ISSN No: $2456-6470$ | www.ijtsrd.com | Volume - 2 | Issue -4 \\
\hline
\end{tabular}

\title{
Analysis on The Impact of Reflectance in Optical Fiber Links
}

\author{
J. Ilouno ${ }^{1}$, M. Awoji ${ }^{2}$, J. Sani ${ }^{3}$ \\ ${ }^{1}$ Physics Department, University of Jos, Jos, Nigeria \\ ${ }^{2}$ Physics Department, Kwararafa University, Wukari, Taraba State, Nigeria \\ ${ }^{3}$ Backbone Connectivity Network, Abuja, Nigeria
}

\section{ABSTRACT}

An optical fiber link is a part of an optic fiber communication system. Other components of the optic fiber link include the transmitter, connectors, and the receiver. The optical fiber could be singlemode (for long distance transmission) or multi-mode (for short distance transmission). This paper however, majors on the impact of reflectance in the single-mode optical fiber. Reflectance is a hidden threat that increases Bit Error Rate, BER, (rate at which errors occur in transmission system) and reduces system performance if not monitored or controlled. Optical Time Domain Reflectometer (OTDR) was used to measure the reflectance in single-mode fiber. Events measurements in OTDR heavily depend on good reflectance. The OTDR was able to establish the reflectance in every portion of the fiber under test. An average reflectance level of $-14.9275 \mathrm{~dB}$ of $1550 \mathrm{~nm}$ signal over the span length of $20.422 \mathrm{~km}$ was achieved which is within the acceptable standard range. Hence, good quality performance transmissions can be achieved along these routes.

Keywords: Reflectance, OTDR, Fiber, Optical Fiber Link

\section{INTRODUCTION}

Fiber optic or optical fiber link comprises of optical fiber, a transmitter, a receiver and connectors as seen in Figure 1[1].

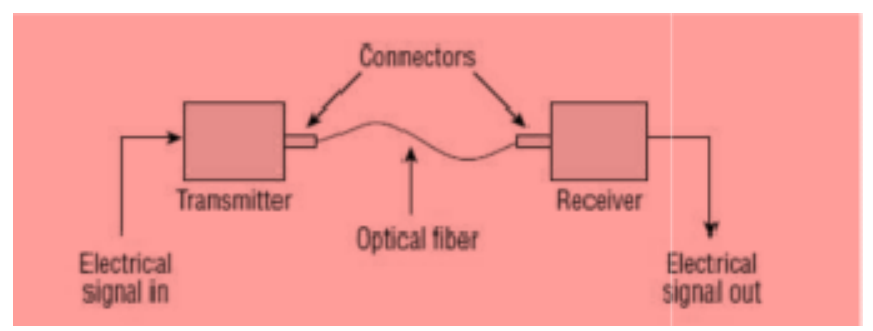

Figure 1: Optical fiber link
The two main function of the transmitter are [2]: it serves as a light source launched into the optical fiber cable and to modulate the light by the binary data it receives from the source. The receiver has two functions: it detects the light coupled out of the optical fiber cable and converts the light into electrical signal, and demodulates the light to determine the identity of the binary data that it represents. The connectors are used to attach the optical fiber and provide solid contact between mated transmitter and receiver. The connector must align the optical fiber end precisely with the light source or receiver to prevent signal loss An optical fiber is a long thin strand of pure glass used as the transmission medium for the signal [3]. It comprises of two concentric layers called the core and the cladding with different refractive indexes as shown in Figure 2 [4]. The index of refraction is a way of measuring the light in a material. Fiber could be multi-mode (suitable for short-haul, limited bandwidth and relatively low-cost applications) or single-mode (suitable for highbandwidth and medium- and long-haul applications). One of the major considerations with all types of fiber-fiber connection is the optical loss encountered at the interface.

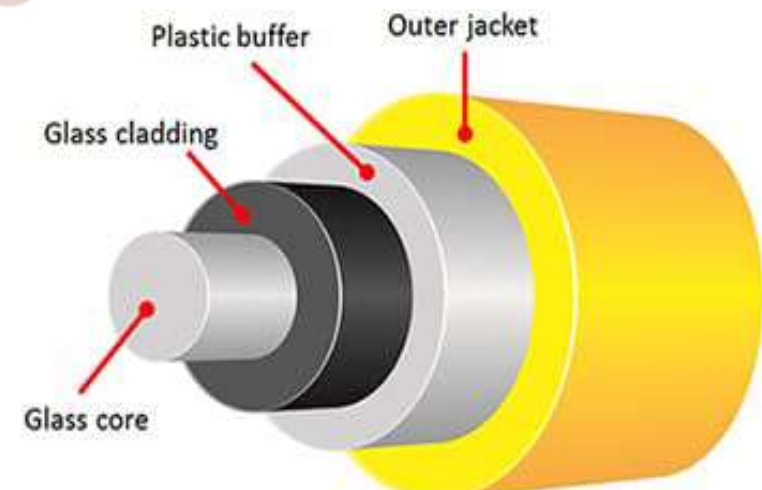

Figure 2: Optical fiber structure 
Even when the two jointed fiber ends are smooth and perpendicular to the fiber axes, and the two fiber axes are perfectly aligned, a small proportion of the light may be reflected back into the transmitting fiber causing attenuation at the joint. This phenomenon is known as Fresnel reflection and is associated with the step changes in refractive index at the jointed interface (i.e. glass-air-glass) that leads to reflectance [5].Reflectance is the amount of light reflected from a single discontinuity in an optical fiber link such as a connector pair or the percentage of light reflected by a single component $[1,2]$. Reflectance is expressed as the ratio of the intensity of light reflected to the incident light intensity in $\mathrm{dB}$ [6]. Light traveling down the fiber when it sees a change in refractive index, then reflection (reflectance) occurs. The most common causes of reflectance may arise from air gap between the connectors and dirt or residue left behind by the cleaning solution [7]. Connectors possess different ferrule end finishes to minimize reflectance as well as loss. Reflectance of low values is obtainable from fusion splicing and from careful designed mechanical joints. However, certain mechanisms can cause larger values of reflectance. These include optical interference produced in the cavity between two fiber end faces as well as reflection from a high-index layer formed on the end face of a highly polished fiber. Typical reflectance measurements require a large dynamic range. Accepted reflectance values in the optical telecommunication industry vary according to the connector type as seen in Table 1 [8]:

Table 1: Typical connectors with their reflectance

\begin{tabular}{|l|l|}
\hline Connector Type & Typical Reflectance \\
\hline Flat with air & $-20 \mathrm{~dB}$ \\
\hline Physical Contact (PC) & -30 to $40 \mathrm{~dB}$ \\
\hline Ultra PC & -40 to $50 \mathrm{~dB}$ \\
\hline APC (angled) & $-60 \mathrm{~dB}$ or higher \\
\hline
\end{tabular}

For laser based systems, reflectance has the capacity to reduce system performance which affects the stability of the laser source. Also, in systems with more than a connector multiple reflections occur and this increases the level of signal noise at the optical detector. The specific component at fault can be identified with optical equipment such as an Optical Time Domain Reflectometor, OTDR.
An OTDR can be used in the measurements of reflectance in optical fiber. A typical OTDR consists of eight basic components: the directional coupler, laser generator, time circuit, signal-board computer, Digital Signal Processor (DSP), and analogy to digital converter, sample-and-hold circuit, and avalanche photodiode as shown in Figure 3 [9].

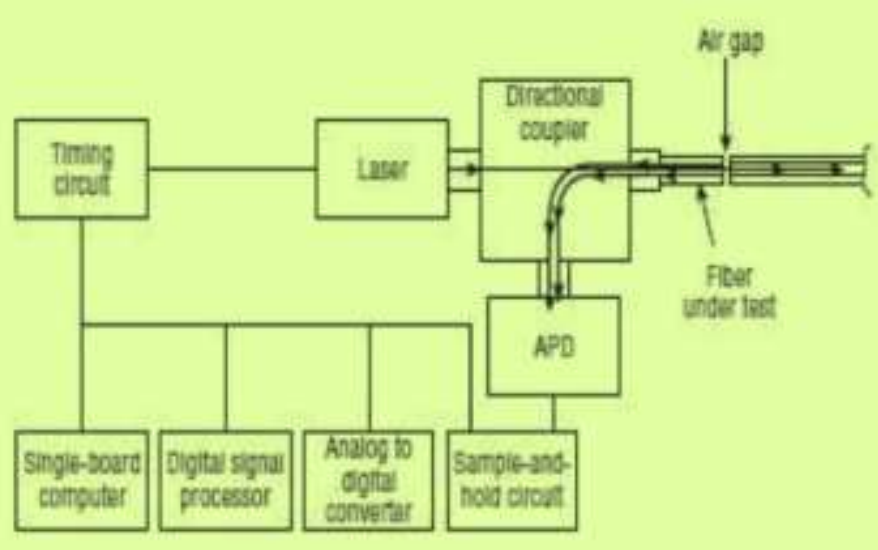

Figure 3: Block diagram of OTDR

Figure 3 shows how light pulses are launched from the laser through the directional coupler into the optical fiber. The directional coupler channels light returned by the optical fiber to the avalanche photodiode. The avalanche photodiode then converts the light energy into electrical energy. The electrical energy is sampled at a very high rate by the sampleand-hold circuit. The sample-and-hold circuit maintains the instantaneous voltage level of each sample long enough for the analogy to digital converter to convert the electrical value to a numerical value. The numerical value from the analogy to digital converter processed by the DSP and the result is sent to the single-board computer to be stored in memory and displayed on the screen. The entire process is typically repeated many times during a single test of an optical fiber and coordinated by the timing. The OTDR will send the light constantly during certain period. The OTDR capture each sample in round-trip time means the actually transmitting time is half of what the OTDR counts. The OTDR shows the time or distance on the horizontal axis and amplitude on the vertical axis. The horizontal axis's unit is shown in meters or kilometers, and $\mathrm{dB}$ (decimal) in vertical axis. The trace generated by the OTDR can be used to identify the reflectance as seen in Figure 4. High peak indicates high reflectance. Limits may occur to the dead zone (i.e. the distance or time where OTDR cannot detect or precisely localize event on the fiber) of the OTDR. However, dead zones boxes that 
comply with TIA-455 (standard test procedures for fiber optics components) allow reflectance measurements to be made within the OTDR's deadzone [7].

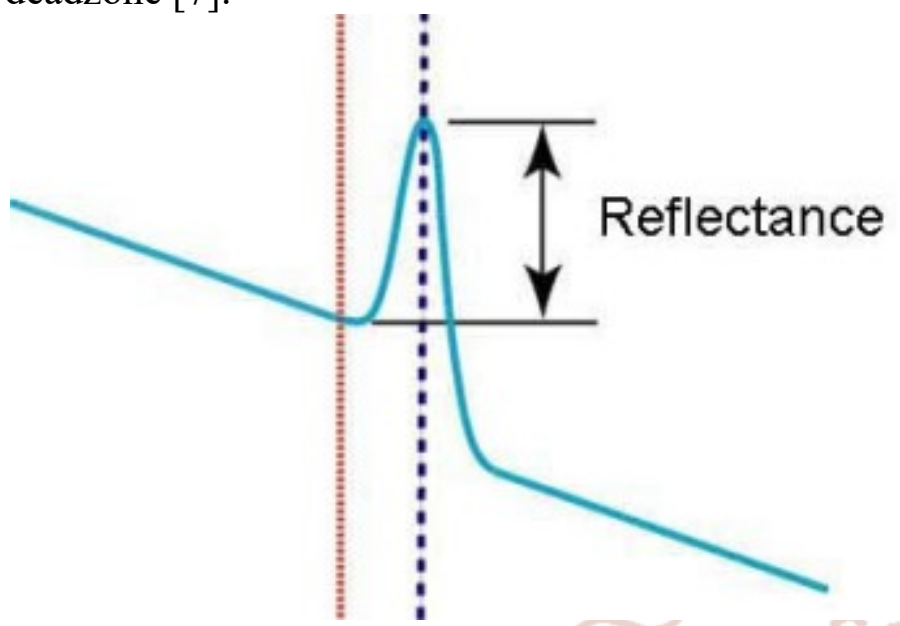

Figure 4: Reflectance

Figure 4: OTDR Trace indicating Relectance

\section{MATERIALS \& METHODS}

\section{Materials:}

1. Single-Mode Patch cords

2. Power meter

3. Optical Time Domain Reflectometer (OTDR)

4. Media Converter/Transmission Equipment

5. Flash drive

OTDR test procedures

Fiber Type: SM 96 CORE FIBER STERLITE

Device: MTS 6000 Num.2487

Module: 8126LR Num.16131

The OTDR parameters were set as:

Wavelength: $1550 \mathrm{~nm}$

Range (Km): 20.422

Acq. Time: 20 s

Resolution: $64 \mathrm{~cm}$

Index: $\quad 1.46800$

A power meter was used in testing for continuity along the cable before the measurements were taken. A single-mode patch cord was attached to the OTDR and to cable plant (core 01) under test via the patch panel at point A as shown in Figure 5. The OTDR was preset manually as stated above and it emitted light power pulses along the cable in a forward direction by the injection laser. The light pulses then bounced back and were measured by the factoring out of time and distances. The backscattered light was detected by the Avalanche photodiode receiver. The output of the photodiode receiver was driven by an integrator which improved the Signal to Noise Ratio (SNR) by giving an arithmetic average over a number of measurements at one point. This signal was fed into a logarithmic amplifier and the average measurements for successive points within the fiber were plotted and recorded with the chart recorder. The media converter was then used in converting the trace to readable format and retrieved with an external drive. The same procedure was repeated for cores 02 to 24 of the fiber and results tabulated as seen in Table 2 .

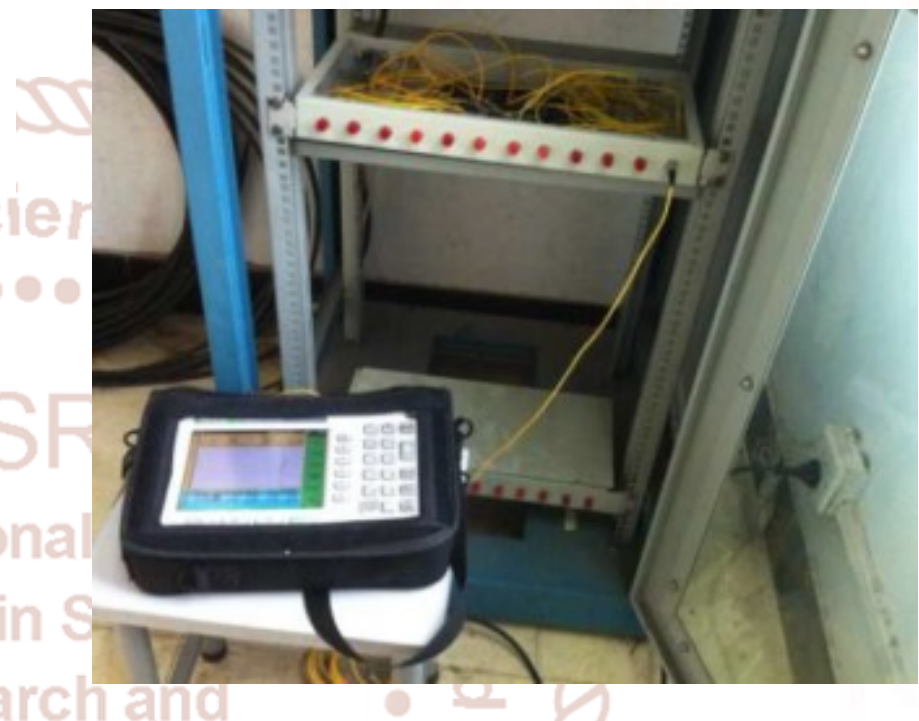

Figure 5: OTDR connected to fiber under test

III. RESULTS

Table 2: Reflectance levels

\begin{tabular}{|c|r|}
\hline Fiber No. & \multicolumn{1}{|c|}{$\begin{array}{l}\text { Reflectance } \\
(\mathrm{dB})\end{array}$} \\
\hline 1 & -16.02 \\
\hline 2 & -16.92 \\
\hline 3 & -14.85 \\
\hline 4 & -17.16 \\
\hline 5 & -16.83 \\
\hline 6 & -17.16 \\
\hline 7 & -17.37 \\
\hline 8 & -16.54 \\
\hline 9 & -16.41 \\
\hline
\end{tabular}


International Journal of Trend in Scientific Research and Development (IJTSRD) ISSN: 2456-6470

\begin{tabular}{|c|c|}
\hline 10 & -17.09 \\
\hline 11 & -17.14 \\
\hline 12 & 17.28 \\
\hline 13 & -16.20 \\
\hline 14 & -17.00 \\
\hline 15 & -16.89 \\
\hline 16 & -16.87 \\
\hline 17 & -16.88 \\
\hline 18 & -16.85 \\
\hline 19 & -17.16 \\
\hline 20 & -14.83 \\
\hline 21 & -14.74 \\
\hline 22 & -14.61 \\
\hline 23 & -15.15 \\
\hline 24 & -14.87 \\
\hline
\end{tabular}

\section{REFERENCES}

1) G.P. Agrawal, Fiber-optic communication systems (New York: John Wiley \& Sons ISBN 047121571-6, 2002).

2) W. Etten \& J. Plaats, Fundamentals of optical fiber communications (New York: Prentice Hall, 1991).

3) S. Dharamvir, Optical fiber based communication network, International Journal of Technical Research (IJTR), 1 (1), 2012, 43-46.

4) https://www.vialite.com/resourses/guides/singlemode-vs-mult-mode/ (2018)

5) M. Ilyas \& H.T. Moftah, Handbook of optical communication networks (Florida, USA: CRC Press, 2003).

6) http://questtel.com/wiki/fiber-return-loss-andreflectance (2018)

7) J.M. Senior, Optical/Fiber Communications Principles and Practice, 3rd Edition (England: Pearson Practice Hall, 2009)

8) http://www.thefoa.org/tech/ref/testing/test/reflecta nce.html (2018)

9) S.K. Raghuwanshi, Experimental characterization of fiber optic communication link for digital transmission system. ICTACT Journal on Communication Technology, 5 (10), 2014, 868876.

An average reflectance level of $-14.9275 \mathrm{~dB}$ was obtained which is within the standard specification. These small reflection events did not pose any threat or risk to the transmission capability or mechanical reliability. Therefore, it is not cost effective or advantageous to remove the fibers with these small reflection events since they have little or no impact on the fiber attenuation. The small reflection recorded might have been caused by localized changed in the refractive index of the light carrying portion of the optical fiber. However, measuring more than a 40 to $60 \mathrm{~dB}$ range poses a serious challenge or threat to the performance of an optical fiber link so must be technically avoided.

\section{CONCLUSION}

The impact of reflectance on single-mode fiber optics was analyzed using Optical Time Domain Reflectometer (OTDR). Small reflection events were measured and recorded as reflectance which poses no serious challenge to the system performance because their impact on the fiber attenuation was minimal or zero. 